\title{
Zur Verwendung nationaler Identitätskomponenten in der Unternehmenskommunikation - ein deutsch-dänischer Vergleich
}

\author{
Aase Voldgaard Larsen, Anne Grethe Julius Pedersen und Katrine Knudsen (Aalborg)
}

\begin{abstract}
How does globalization influence companies' depiction of their national origin? In this study, we analyse and compare corporate websites of German and Danish companies related to the wind energy sector. Traditionally, Country of Origin (COO) has been used within certain industries with the purpose of achieving a competitive advantage. The wind energy sector is a relatively new sector with market potential worldwide. This situation has led us to investigate whether companies in this sector regard themselves as national and whether they choose to position themselves by means of $\mathrm{COO}$ indicators. The analysis includes various indicators of national affiliation, such as the use of national flags or colours and references to Germany or Denmark. Moreover, we investigate which alternative parameters are chosen by the companies that do not depict themselves through national identity features. In summary, the study shows that the national identity is still of great importance and is regarded as a valuable position parameter in the cleantech-industry.
\end{abstract}

\section{$1 \quad$ Einleitung}

Der vorliegende Beitrag beschäftigt sich mit dem Spannungsfeld zwischen Globalisierung und nationaler Identität im Bereich der Unternehmenskommunikation. Es wird der Frage nachgegangen, wie sich die intensivierte Globalisierung aktuell auf das dargestellte Selbstbild von deutschen und dänischen Unternehmen auswirkt. Dabei wird untersucht, inwieweit Selbstdarstellungen auf Corporate Websites von einer Betonung der nationalen Herkunft geprägt sind. Die Identität eines Unternehmens ist eine komplexe Größe, die sich nicht in einer Studie wie dieser vollständig erörtern lässt. Dargestellte Unternehmensidentitäten können aber als ein Indikator dafür betrachtet werden, ob sich Unternehmen eher als global players verstehen und auf eine Betonung der nationalen Zugehörigkeit verzichten oder die nationale Zugehörigkeit als Teil ihrer Identität hervorheben. Die Entscheidungsträger in Management und Marketing stehen vor dieser grundlegenden Wahl, die von Faktoren wie beispielsweise Produkt, Bekanntheit und Wettbewerbssituation abhängen kann, aber auch von den - konkreten und diffuseren - Vorstellungen über das eigene Land und andere Länder. Traditionell hat die Lebensmittelindustrie in Dänemark und die Automobilindustrie in Deutschland nationale Identitätskomponenten als Positionierungsparameter benutzt, aber wie stellt sich eine relativ neuetablierte Branche wie die Cleantech-Branche zu dieser Frage? Das wird im Folgenden untersucht. Durch einen solchen Einblick in die nach außen hin kommunizierten Selbstbilder

Linguistik online 79, 5/2016 - http://dx.doi.org/10.13092/lo.79.3343

CC by 3.0 
von Unternehmen erfahren wir mehr darüber, wie sich die Globalisierung auf den Nationalitätsbezug in der Unternehmenskommunikation auswirkt und dadurch auch, wie wir allgemein den Einfluss der Globalisierung auf das gesellschaftliche Leben zu beurteilen haben.

\section{Unternehmensidentität in einer globalisierten Welt}

Unter Globalisierung verstehen wir einen Prozess, der sich mit dem Zusammenbruch des Ostblocks und der darauf folgenden Öffnung der Grenzen zwischen Ost und West Ende der 1980er und Anfang der 1990er Jahre intensiviert hat (cf. Giese/Mossing/Schröder 2011: 9). Nach Ulrich Beck (1998) umfasst die Globalisierung ,die Prozesse, in deren Folge die Nationalstaaten und ihre Souveränität durch transnationale Akteure, ihre Machtchancen, Orientierungen, Identitäten und Netzwerke unterlaufen und querverbunden werden“ (1998: 28-29). Durch zunehmend erweiterte zwischengesellschaftliche Beziehungen entwickelt sich ein weltumspannendes Netzwerk (cf. Brock 2008: 13), wobei vor allem Handelsbeziehungen eine zentrale Rolle spielen. Aus wirtschaftlicher Sicht bedeutet die Globalisierung vor allem ein System internationaler Arbeitsteilung und Spezialisierung sowie globale Arbeits- und Kapitalmärkte. Auf Unternehmensebene hat der Globalisierungsprozess u. a. dazu geführt, dass sich viele Unternehmen zu sogenannten global players entwickelt haben, die nicht allein ihre im Heimatland produzierten Waren exportieren, sondern auch ihre Produktionsaktivitäten, Kompetenzen und Koordinationsaufgaben international ansiedeln (cf. Giese/Mossing/Schröder 2011: 16-19; Brock 2008: 53).

Mit dem Prozess der Globalisierung verstärkt sich generell das Bewusstsein, dass alle Menschen in einer gemeinsamen Welt leben. Dem gegenüber steht aber eine Tendenz der erhöhten Aufmerksamkeit für das, was Menschen unterscheidet, so z. B. nationale Merkmale (cf. Jensen 2013: 127). Übertragen auf Unternehmen stellt sich die Frage, wie die veränderten gesellschaftlichen Rahmenbedingungen ihre Identität und Strategie beeinflussen. Dem hier zugrunde gelegten prozessualen Verständnis von Globalisierung zufolge lässt sich das Spannungsfeld zwischen Nationalität und Globalität jedoch nicht als ein absolutes Entweder-oder begreifen, sondern vielmehr als eine Selbstwahrnehmung, die eher national oder global fundiert ist.

\subsection{Der Begriff der Identität}

In manchen Darstellungen wird der Begriff Identität fehlerhaft als ,,something that simply $i s^{\text {“ }}$ (Jenkins 2004: 5; Hervorhebung im Original) beschrieben. Das heißt also, er wird als eine stabile Eigenschaft (a ,thing“) dargestellt, die stationär und unveränderlich ist. Korrekter ist es aber Identität „not as a thing but a process“ (Mole 2007: 10; Hervorhebung im Original) zu begreifen, denn es handelt sich um einen aus flüssigen Elementen bestehenden, dynamischen, unter ständiger Veränderung befindlichen und durch Interaktion konstruierten Prozess (cf. Jenkins 2004: 5; Mole 2007: 10). Wichtig ist dabei auch, dass eine Person oder eine Gemeinschaft nicht nur eine Identität hat, sondern dass sich beispielsweise bei jeder Person ständig eine Pluralität von Identitäten in der Entwicklung befindet (cf. Jenkins 2004: 5), da sie oder er mehrere soziale Rollen in unterschiedlichen Gruppen ausfüllt. Mit dieser Auffassung von Identität geht einher, dass Identität ein sozial konstruiertes Phänomen ist, das durch sprachliche und visuelle Mittel in einem bestimmten Kontext realisiert wird. Es herrscht somit 
Nationale Identitätskomponenten in der Unternehmenskommunikation

weitgehend Übereinstimmung darüber, dass die Identität einer Person fließend ist und sich nach den Umständen und den Möglichkeiten formt und ändert (cf. Kabir 2013: 23). Bei der Bestimmung von Identität sieht Jenkins (2014) die Konstruktion von Gleichheiten und Unterschieden zwischen verschiedenen Individuen oder Gemeinschaften als bedeutungsvoll an. Es handelt sich dabei um die gemeinsame Zugehörigkeit (cf. Gilroy 1997: 301-302) und die Gemeinsamkeiten der Gruppenmitglieder (cf. Kabir 2013: 24), sowie gleichzeitig um die Unterschiede der Gruppe zu anderen.

Was die Zuschreibung von Identität anbelangt, sind in der Forschung so genannte ,identity markers“" aufgezeigt worden. Dazu gehören u. a. Geburtsort, Verwandtschaft, Wohnort, Zeitraum des Wohnens, Erziehung und Ausbildung, Name, physische Erscheinung und Zugehörigkeit zum Ort (cf. Kiely et al. 2001: 36) sowie nationale, ethnische oder regionale Zugehörigkeit. Daraus wird deutlich, dass identitätsbildende Merkmale mit Ort, Zeit und physischer Erscheinung eng verknüpft sind. Identität bezieht sich aber nicht nur auf Ortszugehörigkeit und Nationalität. Sie kann ein Bewusstseinszustand, ein „state of mind“, sein (Douglas 2009: 19 - zit. nach Devine/Logue 2002: 264). Die Identität ist für den einzelnen wichtig, da sie ihm die Beantwortung der Frage „Wer bin ich?“ erleichtert, und gleichzeitig hilft sie uns, die Welt in überschaubare Abschnitte einzuteilen und in der Beziehung zur Welt Sinn zu generieren (cf. Gilroy 2000: 98). Wichtig ist außerdem, dass die kontextuellen Umstände den Rahmen für die Entwicklung der Identität bilden (cf. Bamberg/De Fina/Schiffrin 2011). Übertragen auf die unternehmerische Identitätsbildung heißt das, dass u. a. die Globalisierung und ihre Auswirkungen eine mitgestaltende Rolle dabei spielen, was geäußert wird und mit welchen verbalen und visuellen Mitteln.

\subsection{Unternehmensidentität}

Auch für Unternehmen ist eine konsistente, kontinuierliche, wenn auch langfristig unter ständiger Veränderung stehende Identität (cf. Herbst 2012: 31) nicht zuletzt in der globalisierten Welt von großer Bedeutung. Produkte und Dienstleistungen sind weltweit zugänglich, was den Wettbewerbsdruck und auch die Ähnlichkeit und Austauschbarkeit vieler Produkte und Dienstleistungen erhöht. Qualität ist selbstverständlich geworden und ermöglicht „kaum noch Unterscheidung von Konkurrenten“ (Herbst 2012: 12). Folglich spielt auch Vertrauen zu Unternehmen bei der Kaufentscheidung eine immer größere Rolle, nicht nur im B2C-Bereich, sondern auch im B2B-Geschäft. Vertrauen kann im Marketing-relatierten Zusammenhang so definiert werden, dass der Kunde sich freiwillig darauf verlässt, dass die Marke bzw. der Anbieter fähig und bereit ist, eine gegebene Leistung zu erfüllen, um so den Erwartungen des Kunden gerecht zu werden (cf. Meffert/Burmann/Kirchgeorg 2012: 132). Anbieter können gezielt versuchen, das Vertrauen der Kunden zu erhöhen, indem sie auf Qualitätsmanagement und Kompetenzbeweise durch z. B. Testinstitute, Zertifizierungen und Referenzen sowie auf Aufbau der Marke (cf. Meffert/Burmann/Kirchgeorg 2012: 132) setzen, d. h. auch durch ihre Markenarbeit die Identität stärken. Sympathie allein ist „kaum geeignet, sich eindeutig und dauerhaft gegenüber anderen Unternehmen abzugrenzen“, stattdessen müssen „einzigartige Gefühle“ mit dem Unternehmen verbunden werden (Herbst 2012: 48). Dadurch kann sich das Unternehmen gegenüber den Kunden identifizieren, profilieren und von anderen Wettbewerbern differenzieren. Dies sind die Ziele der Unternehmensidentität (cf. Birkigt/Stadler/Funck 
2002). Wenn sich Unternehmen in den Augen der Bezugsgruppen von anderen Unternehmen unterscheiden wollen, ist die Unternehmensidentität so zu kommunizieren, dass diese als einzigartig erscheint.

So beschreibt z. B. Kückelhaus (1998: 356) im Folgenden präzise, warum Unternehmen zunehmend so viel Arbeit in den Aufbau einer einheitlichen Unternehmensidentität investieren: „Konsequenzen einer schlüssigen Corporate Identity sind Vertrauen, Glaubwürdigkeit, Bekanntheit und Kompetenz bezüglich der Leistungserstellung im Markt und bei der internen und externen Öffentlichkeit“" (cf. auch Birkigt/Stadler/Funck 2002: 48 und Herbst 2012: 79, die Sicherheit hinzufügen). Auf diese Weise kann das Unternehmen aus der Informationsflut heraustreten, erkennbar werden und dadurch das Verhältnis zu seinen Bezugsgruppen stabilisieren. Die Unternehmensidentität verkörpert die Persönlichkeit des Unternehmens. Die Gestaltung der Unternehmenspersönlichkeit setzt sich nach Herbst (2012) aus den Elementen Kultur, Leitbild, Image und Instrumenten (d. h. Corporate Design, Corporate Communication, Corporate Behaviour) zusammen (cf. Herbst 2012: 88). In anderen Darstellungen werden mehr, weniger oder andere Elemente mit einbezogen oder unterschiedlich zueinander in Verbindung gesetzt, so wird z. B. bei Birkigt/Stadler/Funck (2002) nur Unternehmensdesign, kommunikation und -verhalten zur Unternehmenspersönlichkeit gezählt, während z. B. das Fremdbild (Image) als Projektion der Identität in den öffentlichen Raum aufgefasst wird. Bei Melewar/Karaosmanoglu (2006) werden gleichrangig neben die von Herbst „Instrumente“ genannten Elemente auch Corporate Culture, Corporate Strategy, Corporate Structure und Industry Identity gestellt (2006: 849), bei Melewar/Jenkins (2002) werden jedoch nur die Elemente Corporate Design, Corporate Communication, Corporate Behaviour, Corporate Culture und Market Conditions mit einbezogen (2002: 81). Hatch/Schultz (1997) sehen Organisationskultur als ,a context within which interpretations of organizational identity are formed and intentions to influence organizational image are formulated“ (Hatch/Schultz 1997: 357), nicht als einen Teil der eigentlichen Unternehmensidentität. In diesem Beitrag wird Unternehmenskultur, und besonders Country of Origin (COO), als Teil der Unternehmensidentität aufgefasst und untersucht. Dabei erscheint von geringerer Bedeutung, ob die Elemente des Corporate Identity gleichrangig zueinander angeordnet, oder als einander untergeordnete Elemente gesehen werden. COO wird in diesem Beitrag als Teil der Unternehmenskultur und damit als Teil der Unternehmenspersönlichkeit aufgefasst (cf. z. B. auch die Diskussion der unterschiedlichen Definitionen von Corporate Identity bei Melewar/Jenkins 2002).

Ein Unternehmen hat immer eine Unternehmenskultur; „es ist nicht möglich, dass es keine gibt“" (Herbst 2012: 90). Diese Kultur gestaltet die Unternehmenspersönlichkeit immer in großem Umfang mit, da es sich dabei um die Werte und Normen des Unternehmens handelt. Unternehmensidentität ist dabei die strategische Manifestation der Vision und Mission unterstützt von den im Management und in der Produktion durch das Unternehmen eingesetzten Strategien (cf. Melewar/Wooldridge 2001). Von großer Bedeutung sind dabei die ethischen und kulturellen Werte sowie Geschichte und Philosophie des Unternehmens (cf. Ind 1992; zit. nach Melewar/Karaosmanoglu 2006: 847-848).

Die Bezugsgruppen des Unternehmens bilden sich anhand der identitätsstiftenden Elemente eine Auffassung oder ein Bild von dem Unternehmen, das Image (cf. Melewar/Karaosmanoglu 2006: 848). Es handelt sich dabei allerdings nicht einfach um eine Pro- 
Nationale Identitätskomponenten in der Unternehmenskommunikation

jektion oder ein Spiegelbild der Unternehmensidentität in der Außenwelt, wie es von Birkigt/Stadler/Funck (2002: 23) gesehen wird. Vielmehr wird das Unternehmensimage von anderen Stimmen als denen des Unternehmens mitbeeinflusst. Ein starkes Image stärkt die Kundenbeziehung und hat damit eine Schutzfunktion für das Unternehmen (cf. Saldsieder/Saldsieder 2010: 189).

\section{Nationale Identitätskomponenten in der Unternehmenskommunikation}

Die Verwendung von nationalen Identitätskomponenten in der Unternehmenskommunikation hat eine lange Tradition. Das Interesse für das Ursprungsland eines Produkts hat seine Wurzeln im 19. Jahrhundert mit einem britischen Gesetz, das mit protektionistischem Ziel britische Produkte durch ein Verbot der fälschlichen Kennzeichnung ausländischer Produkte als britisch schützen sollte (cf. Head 1988: 237). Dies galt u. a. für Messer und andere Stahlerzeugnisse aus Deutschland. Das als negative Kennzeichnung gedachte ,Made in GermanyLabel` wurde mit der Zeit aber ein Qualitätszeichen, das von den „Herstellern der qualitativ hochwertigen und zuverlässigen Produkte“ als „Markierungsinstrument und Verkaufsargument" eingesetzt werden konnte (Nielsen 2005: 156).

Heute wird COO als Instrument der Positionierung und Differenzierung am Markt benutzt, um Produkte und Unternehmen positiv mit ihrem Ursprungsland zu kennzeichnen. Ein nationales Image baut auf einem kognitiven Netzwerk auf, in dem gelagertes Wissen, Werte und Haltungen ein Netzwerk aus Assoziationen über ein Objekt bilden (cf. Djursaa/Kragh/Møller 1991: 124; Niss 1994: 34-35). Bekannte Beispiele für die Verwendung des Herkunftslandes als zentralem Element der Vermarktung sind aus Dänemark Lurpak, die Buttermarke mit den Luren und der Aufschrift Dänische Butter, Dänisches Bettenlager, Danish Crown und die Marmelade von Den Gamle Fabrik. Aus Deutschland gehören $V W$ mit dem auch in Dänemark verwendeten Slogan Das Auto, Rittersport sowie Germanwings dazu. Dabei wird angenommen, dass auf positive nationalstereotypische Merkmale zurückgegriffen wird, wie z. B. deutsche Gründlichkeit (cf. Herbst 2012: 89) und Effizienz sowie japanische Innovation (cf. Melewar/Karaosmanoglu, 2006: 856). Eine Untersuchung zeigte 1992, dass Deutschland mit Zuverlässigkeit, Stabilität, Qualität und Service, aber nur wenig mit Design, Style und attraktiven Preisen verbunden wurde (cf. Jaffe/Nebenzahl 2006: 83). Niss (1996: 14) bemerkt, dass „Made in Denmark“ hohe Qualität und Innovation in der Produktentwicklung und im Design konnotiert. Bei diesen Zuschreibungen von bestimmten Merkmalen ist aber von einem Wechselspiel die Rede, indem die Produktmerkmale gleichzeitig auch die Vorstellungen vom Ursprungsland mitgestalten.

Nicht nur das „Made in“-Konzept hat eine Bedeutung, sondern auch Marken- und Unternehmensnamen, Top-Level-Domains, Slogans usw. (cf. Papadopoulos 1993: 14-15) können Aussagen über die Zugehörigkeit eines Unternehmens zu einem bestimmten Land machen. Nielsen (2005) hat z. B. die dänischen Buchstaben $\propto$, ø, å sowie die Namensgebung anhand subtraktiver Wortbildung mit Dan und Scan und des Patronymikons -sen untersucht. Singh/Pereira (2005) haben eine Liste von 36 Merkmalen unterschiedlichster Natur zusammengestellt, die auf Websites vorkommen und die Kulturdimensionen von Hofstede (1980) und Hall (1976) reflektieren können. Zu diesen Merkmalen gehören z. B. Personalisierung, Informationen zur Unternehmenshierarchie, Freundlichkeit, Verkaufsbedingungen, Symbole 
und Bilder der nationalen Identität, Traditionsthema (darunter Betonung der Geschichte sowie Band zum Herkunftsland), Qualitätsversicherung und Awards sowie die Anwendung von Superlativen. Für die in diesem Beitrag erläuterte Forschungsfrage sind jedoch nur diejenigen Merkmale relevant, die Nationalität signalisieren können.

In Melewar/Karaosmanoglu (2006: 856) stellen die meisten in einer Untersuchung befragten Unternehmensvertreter fest, dass COO eine bedeutungsvolle Einwirkung auf die Unternehmenskultur hat, dass die Bedeutung dieses Elements jedoch bei international tätigen Unternehmen geringer ausfällt. Die Befragten geben weiter an, dass die Bedeutung ihrer Meinung nach als Ergebnis der Globalisierung gesunken ist. Bei Melewar/Jenkins (2002) wird allerdings im Gegenteil dazu behauptet, dass mit der erweiterten globalen Tätigkeit der Unternehmen die Rolle der Nationalität des Unternehmens und der damit verbundenen Merkmale wichtiger geworden sei: „As companies expand their global operations, the role of the nationality of the company and its perceived attributes have become more important" (Melewar/Jenkins 2002: 84). Melewar/Karaosmanoglu (2006) schlagen vor, der Frage nach der Bedeutung nationaler Merkmale bei multinationalen Unternehmen nachzugehen. Im vorliegenden Beitrag, wie in anderen Darstellungen, so z. B. bei Rowlinson and Procter (1999) und Varey and Lewis (2000), wird davon ausgegangen, dass es eine Verbindung zwischen der Nationalkultur, aus der das Unternehmen stammt, und der Unternehmensidentität gibt.

COO ist laut Nielsen (2005) auch jetzt noch wichtig, weil sie den Menschen mit der „Orientierung an der Instanz ,Ort““ (in der Bedeutung geographischer Herkunft) hilft, d. h. mit der „Identifikation bzw. Feststellung der eigenen Position in der Relation zur Umwelt und zur Umgebung“ (Nielsen 2005: 155). Bei Nielsen (2005: 166-167) findet man den Entwurf einer Skala, die von der geringen Ausprägung (durch graphemische Sonderzeichen, Patronymika, subtraktive Wortbildungen mit -sen und Domainnamen) bis hin zur starken Ausprägung der COO-Markierung (anhand von Slogans, integrierter Thematisierung der Nationalität und nationaler Markenidentität) geht.

\begin{tabular}{|c|c|c|c|c|c|c|c|}
\hline \multirow[b]{2}{*}{$\begin{array}{l}\text { Ebene der } \\
\text { COO- } \\
\text { Markierung }\end{array}$} & \multicolumn{2}{|c|}{$\begin{array}{r}\text { Geringe Ausprägung der } \\
\text { COO-Markierung }\end{array}$} & \multicolumn{3}{|c|}{ 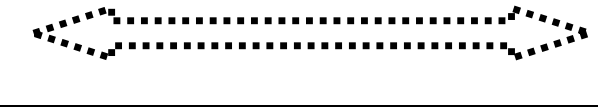 } & \multicolumn{2}{|c|}{$\begin{array}{r}\text { Starke Ausprägung der } \\
\text { COO-Markierung }\end{array}$} \\
\hline & $\begin{array}{l}\text { Graphe- } \\
\text { misch }\end{array}$ & $\begin{array}{l}\text { Onomas- } \\
\text { tisch }\end{array}$ & $\begin{array}{l}\text { Onomas- } \\
\text { tisch }\end{array}$ & Internet & $\begin{array}{l}\text { Werbe- } \\
\text { aussage- } \\
\text { bezogen }\end{array}$ & Integriert & Integriert \\
\hline $\begin{array}{l}\text { Ausdruck } \\
\text { der COO- } \\
\text { Markierung }\end{array}$ & $\begin{array}{l}\text { Sonder- } \\
\text { zeichen }\end{array}$ & $\begin{array}{l}\text { Patro- } \\
\text { nymikon }\end{array}$ & $\begin{array}{l}\text { Subtraktive } \\
\text { Wortbildung }\end{array}$ & $\begin{array}{l}\text { Domain- } \\
\text { Name }\end{array}$ & Slogan & $\begin{array}{l}\text { Thema- } \\
\text { tisierung }\end{array}$ & $\begin{array}{l}\text { Marken- } \\
\text { identität }\end{array}$ \\
\hline $\begin{array}{l}\text { Beispiel im } \\
\text { dänisch- } \\
\text { deutschen } \\
\text { Kontext }\end{array}$ & $a, \varnothing, \stackrel{a}{ }$ & -sen & Dan- & $w w w \ldots d k$ & $\begin{array}{l}\text { Prima, } \\
\text { prima aus } \\
\text { Dänemark }\end{array}$ & $\begin{array}{l}\text { Kleines } \\
\text { Land, } \\
\text { großer } \\
\text { Akvavit }\end{array}$ & $\begin{array}{l}\text { Buch der } \\
\text { Wahrheit }\end{array}$ \\
\hline
\end{tabular}

Tabelle 1: Skala der Gestaltungsmöglichkeiten der COO-Markierung (Nielsen 2005: 166)

Die Herstellungsprozesse werden immer komplexer, da ,multinationale Unternehmen nun ihre Produkte in aller Welt herstellen lassen“ (Nielsen 2005: 157). Manchmal erfolgt z. B. aus Kostengründen ein Outsourcing von der Herstellung der Produkte, während das Design im Ursprungsland bleibt, manchmal werden nur Komponenten an verschiedenen ausländischen Produktionsorten hergestellt, 
Nationale Identitätskomponenten in der Unternehmenskommunikation

während die Produkte im Ursprungsland zusammengebaut werden. Hybride Produkte bilden also eine besondere Herausforderung bei der Verwendung von COO im Marketing. Zur Veranschaulichung dieser teilweise globalisierungsbedingten Komplexität stellen Jaffe/Nebenzahl (2006: 28-32) eine Taxonomie auf, die bei Nielsen (2005: 158) tabellarisch zu sehen ist:

\begin{tabular}{|l|l|}
\hline HC - Home Country & Land, in dem der Verbraucher seinen permanenten Aufenthalt hat \\
\hline DC - Designed-in Country & $\begin{array}{l}\text { Land, in dem das betreffende Produkt sein Design, seine Formge- } \\
\text { bung erhält }\end{array}$ \\
\hline MC - Made-in Country & $\begin{array}{l}\text { Land, dessen Name im „Made in“-Label auftritt, also gleichzuset- } \\
\text { zen mit dem ursprünglichen „klassischen“ COO }\end{array}$ \\
\hline PC - Parts-made-in Country & $\begin{array}{l}\text { Land, in dem Teile/ Komponenten eines Produktes hergestellt wer- } \\
\text { den }\end{array}$ \\
\hline AC - Assembled-in Country & Land, in dem das Produkt gefertigt, zusammengefügt wird \\
\hline OC - Country of Origin & Land, das der Verbraucher mit dem Produkt assoziiert \\
\hline
\end{tabular}

Tabelle 2: COO-Taxonomie nach Jaffe/Nebenzahl (2006: 28-32) und Nielsen (2005: 158)

Diese allerdings nicht ganz konsequente Taxonomie stellt die Komplexität der Design- und Produktionsverhältnisse in der heutigen globalisierten Wirtschaft dar und bietet eine realistische Alternative zur Ablehnung des COO-Begriffes an, indem eine „Abtönung und Differenzierung“ (Nielsen 2005: 158) des Begriffes realisiert wird.

\section{Vorgehensweise und Textkorpus}

Vor dem theoretischen Hintergrund des COO-Ansatzes wird im Folgenden die aktuelle Marktkommunikation von deutschen und dänischen Unternehmen in den Brennpunkt gestellt. Zweck der Analyse ist es, aufzudecken und zu diskutieren, inwieweit die dargestellte Identität von deutschen und dänischen Unternehmen auf ihren Corporate Websites von nationalen Identitätskomponenten geprägt ist.

\subsection{Analysekategorien}

Die Analyse, die sowohl quantitativ als auch qualitativ angelegt ist, umfasst verbale und visuelle Elemente, die auf Nationalität verweisen. Dabei haben wir uns auf die folgenden vier Kategorien festgelegt, weil diese unmittelbar und deutlich die nationale Zugehörigkeit signalisieren:

\section{Verbale Verweise auf Deutschland oder Dänemark}

Zum Verweis auf die nationale Herkunft gehört die explizite Nennung von dänisch/Dänemark bzw. deutsch/Deutschland, beispielsweise bei der Angabe von nationalen Merkmalen. Eine eventuelle Nennung der regionalen oder lokalen Herkunft wird in dieser Studie nicht einbezogen, weil diese ein anderes Zugehörigkeitsgefühl zum Ausdruck bringt.

\section{Nationalfahne und Nationalfarben}

Die Nationalfahne und ihre Farben gelten als visuell deutliches Symbol von einer Nation, da sie an die nationalen Grenzen gebunden sind, was nicht immer für eine Sprache zutrifft. 


\section{Sprache(n) auf der Website}

Über die Verwendung der Nationalfahne als Symbol für die zur Verfügung stehenden Versionen der Website in verschiedenen Sprachen hinaus signalisiert(/-en) auch die gewählte(n) Website-Sprache(n) an sich, wie das Unternehmen seine Identität in der globalen Welt versteht. Ist allein eine englischsprachige Website vorhanden, lässt dies den Schluss auf ein ,globalisierteres' Selbstverständnis zu, als wenn die Webpräsenz (auch) eine dänischsprachige bzw. deutschsprachige Website umfasst.

\section{Internetdomain}

Die Adressen im World Wide Web sind in verschiedene Domains gegliedert, die im letzten Teil der Internetadresse erkennbar sind. Die Internetdomain, auch Top-Level-Domain genannt, kann entweder auf ein Land hinweisen (z. B. .de für Deutschland) oder auf eine Funktion (z. B. .com für kommerzielle Unternehmen oder .org für nichtkommerzielle Organisationen). Mittlerweile sind diese Domains freigegeben worden und für jeden frei zugänglich (Wikipedia 2015).

$\mathrm{Zu}$ bemerken ist, dass die gewählten Analyseschwerpunkte nur eine kleine Auswahl potentieller nationaler Identitätskomponenten umfasst (cf. Abschnitt 3), weshalb kein Anspruch auf Vollständigkeit erhoben wird. Außerdem besteht eine gewisse Überlappungsgefahr zwischen den Kategorien, weil sie oft nicht isoliert funktionieren, sondern in einem Zusammenspiel auftreten. Um einen Überblick zu gewinnen scheint diese Einteilung jedoch sinnvoll.

\subsection{Textkorpus}

Die gewählten Websites stammen wie einleitend erwähnt aus der Cleantech-Branche, da diese in beiden Ländern als eine stark positionierte und vielversprechende Zukunftsbranche gilt. Im Zusammenhang mit der deutschen Energiewende und der dänischen Grøn omstilling [,grüne Umstellung'] spielen die Entwicklung, Produktion und Vermarktung von Umwelttechnologien eine immer größere Rolle in beiden Ländern (cf. z. B. Eksportrådet 2014: 3; Erhvervsog Vækstministeriet 2015; Solvang 2014: 17).

Um das Textkorpus einzugrenzen, wurden dem Ausstellerverzeichnis der WindEnergyMesse 2014 in Hamburg Websites von dänischen und deutschen Ausstellern entnommen (cf. WE 2014). Von den insgesamt rd. 800 deutschen Aussteller-Websites wurden rd. 100 zufällig ausgewählt. Von den dänischen Ausstellern wurden alle, insgesamt rd. 100, Websites analysiert. Die Aussteller vertreten eine breite Palette von Unternehmen der Windindustrie, von Produzenten von Turbinen, über Zulieferer von Ausrüstung, Transporten, Wartung, größeren und kleineren Komponenten bis hin zu Betreibern von Windparks.

\section{$5 \quad$ Ergebnisse der Analyse}

Die analysierten Websites weisen recht unterschiedliche Grade der Signalisierung nationaler Zugehörigkeit auf. Im Folgenden werden die Untersuchungsergebnisse für die deutschen und dänischen Unternehmenswebsites erläutert. 
Nationale Identitätskomponenten in der Unternehmenskommunikation

\subsection{Die deutschen Aussteller-Websites}

Von den insgesamt 100 Websites deutscher Aussteller verweisen 34 explizit auf die deutsche Herkunft oder Platzierung, d. h. etwa ein Drittel der Unternehmen setzt nationale Identitätskomponenten als Positionierungsparameter ein. Bei sechs Unternehmen macht Deutschland oder Germany ein Teil der Firma aus, so z. B. ALSTOM Deutschland AG und ANSYS Germany $\mathrm{GmbH}$; dies ist in den meisten Fällen jedoch nicht Ausdruck dafür, dass es sich um Unternehmen deutschen Ursprungs handelt, sondern um selbständige Abteilungen, z. B. Tochtergesellschaften, von international tätigen Unternehmen. In 23 Fällen wird Deutschland einfach als Standort des Unternehmens angegeben, z. B. Willkommen bei Air Liquide in Deutschland (AIR LIQUIDE Advanced Technologies GmbH), während in elf Fällen die Nationalität als identitätsbildendes Merkmal deutlicher eingesetzt wird. Dabei tritt in drei Fällen die Verwendung des Labels Made in Germany auf (alki TECHNIK GmbH, EDELRID GmbH \& CO. $\mathrm{KG}$, ELA Container Offshore $\mathrm{GmbH}$ ), und in einem vierten Fall ist die Variante Offshoring made in Germany (ALTHOM GmbH) zu sehen. Zudem wird auf einer Website näher erläutert, was das Unternehmen unter ,Made in Germany 'versteht:

(1) alkitronic steht für höchste Ansprüche, langjährige Erfahrung sowie eine kundenorientierte Partnerschaft und verkörpert die klassischen Eigenschaften der „Made in Germany“ Qualität (alki TECHNIK GmbH).

Die genannten Werte höchste Ansprüche, langjährige Erfahrung und kundenorientierte Partnerschaft scheinen hier die mit Deutschland als COO zu verbindenden Eigenschaften zu konkretisieren. Die Bedeutung des deutschen Standorts wird ferner von dem Unternehmen ALT$\mathrm{HOM} \mathrm{GmbH}$ thematisiert, indem hervorgehoben wird, dass die Qualitätssicherung in Deutschland erfolgt und dadurch Quality ensured in Germany zum wichtigsten Versprechen des Unternehmens macht. Ein weiteres Beispiel ist ein quadratischer Label mit der Aussage German quality zur Verknüpfung von Nationalität und Qualität (ADDINOL Lube Oil $\mathrm{GmbH}$ ), und bei mehreren Unternehmen wird die Erfüllung von deutschen Normen oder die ISO-Zertifizierung betont.

Bei den Verweisen auf Deutschland als Produktionsort oder Standort fällt besonders die Aussage des Unternehmens AAT Alber Antriebstechnik GmbH auf:

(2) Der Standort Deutschland ist für AAT ein wesentlicher Bestandteil der Unternehmensphilosophie. Um schnell und flexibel auf Kundenwünsche reagieren zu können, legt AAT Wert auf die Herkunft ihrer Zulieferteile. Daher hat der überwiegende Teil der Lieferanten ihren Sitz in Deutschland, größtenteils sogar in regionaler Umgebung.

Die nationale Zugehörigkeit wird in einigen Fällen mit einem weiteren geographischen Rahmen verknüpft. Ein Unternehmen versteht sich als europäisches und deutsches Unternehmen (Axzion GKS Stahl- und Maschinenbau GmbH), und andere Unternehmen schreiben z. B. deutschland- und europaweit (AllCon Service \& Dienstleistungen $\mathrm{GmbH}$ ) oder auf dem deutschen und internationalen Markt (airwerk $\mathrm{GmbH}$ ). In diesen Fällen erfolgt eine Verknüpfung von der deutschen Herkunft des Unternehmens mit einer internationalen, typisch einer europäischen Identität. Die Slogans auf den untersuchten deutschen Unternehmenswebsites tragen nicht zur landesspezifischen Identität bei, indem sie in keinem Fall Angaben über die Herkunft des Unternehmens enthalten. 
Auf den Websites sind keine deutschen Nationalfahnen zur Thematisierung des deutschen Ursprungs vorhanden, und die Nationalfarben sind bei nur einem Unternehmen als Farbenstriche schwarz-rot-gold im Logo zu sehen (Aquata - Euras Vertriebs- und Produktionsgesellschaft $\mathrm{mbH}$ ). Die Flagge spielt somit bei den untersuchten Websites eine sehr geringe Rolle. Nationalfahnen werden ausschließlich zur Kennzeichnung der Sprachenwahl verwendet, was in 33 Fällen erfolgt. Was die Auswahl an Sprachen angeht, haben elf deutsche Unternehmen ausschließlich deutschsprachige Websites, während sieben nur über englischsprachige Websites verfügen. Ein weiteres Unternehmen betreibt seine Website auf Englisch und Portugiesisch. Die Mehrheit der Unternehmen (80) stellt ihre Websites in mindestens zwei Sprachen zur Verfügung, vor allem Deutsch und Englisch (42). Ein relativ großer Anteil der Websites (26) ist vielsprachig und bietet mindestens zwei weitere Sprachen neben Deutsch und Englisch an, z. B. Chinesisch, Russisch, Französisch, Spanisch, Portugiesisch, Schwedisch und Dänisch. Bezüglich der Internetdomain findet man 55 deutsche Websites mit der Domain de, 38 Websites mit der Domain com und die restlichen sieben Websites mit eu oder anderen Domainbezeichnungen.

Nur drei der 100 deutschen Unternehmen markieren die nationale Identität nicht. Es gibt jedoch auch Beispiele für Unternehmen, die über keine deutschsprachige Website verfügen und com als Internetdomain benutzen, die aber explizit auf deutsch oder Deutschland verweisen, so z. B. founded in Germany, in the center of the European wind energy sector (BaxEnergy $\mathrm{GmbH})$. Sie stellen dadurch einen Bezug zu sowohl Europa als auch Deutschland und dessen Bedeutung für den Windenergiesektor her.

\subsection{Die dänischen Aussteller-Websites}

Auf 41 von insgesamt 99 Websites wird explizit auf die dänische Herkunft verwiesen. Dies erfolgt u. a. durch Aussagen wie wir sind ein dänisches Unternehmen, unser (Haupt-)Sitz liegt in Dänemark oder Danmarks førende [,Dänemarks führendes (Unternehmen)']. Ein auffälliges Beispiel ist die Aussage des Unternehmens Apro Wind A/S, das durch einen Topos der Autorität die nationale Herkunft betont: Danish offshore expertise travels the world. Dies ist ein Zitat des CEO, der mit weißem Schutzhelm und gelber Weste abgebildet ist. Acht Unternehmen führen Danmark oder dansk in ihrer Firma, so z. B. Grene Danmark A/S und Dansk Gummi Industri A/S, auch wenn ihre Websites ansonsten sehr international ausgerichtet sind. Eine besonders explizite Bewertung der dänischen Herkunft ist bei R\&D A/S zu sehen:

(3) Rødderne i R\&D blev sat i 2005, og virksomheden har fortsat forankring i oprindelsen som dansk ingeniørvirksomhed, da vi mener, det danske islæt er et kvalitetsstempel i sig selv [,Die Wurzeln in R\&D wurden 2005 geschlagen und das Unternehmen bleibt weiterhin als dänisches Ingenieurunternehmen mit seinem Ursprung verankert, da wir meinen, dass die dänische Prägung ein Qualitätsstempel an sich ist'].

Was genau mit der dänischen Herkunft zu verbinden ist, bleibt offen, aber u. a. Ingenieurskunst und Expertise scheinen dazuzugehören.

Unter den dänischen Ausstellern auf der WindEnergy Messe war auch die Organisation State of Green, eine öffentlich-private Partnerschaft, die u. a. von der dänischen Regierung und verschiedenen Interessenorganisationen der dänischen Wirtschaft gegründet wurde. Auf deren 
Nationale Identitätskomponenten in der Unternehmenskommunikation

Website, die als Portal für grüne Technologie dient, wird Dänemark als grün und zukunftsorientiert vermarktet. Der Slogan lautet State of Green. Join the Future. Think Denmark. Außerdem betont die Selbstdarstellung auf der Website auch Innovation als Merkmal dänischer Unternehmen:

(4) Danish companies and organisations are at the forefront of green technology and knowhow. State of Green is your gateway to their solutions and to Danish green policies - from green energy to clean water and ressource efficiency.

Außer State of Green und dem Unternehmen A/S Megatrade Beslag treten bei den dänischen Websites keine Slogans auf, die einen Verweis auf dänisch/Dänemark enthalten.

Die rot-weißen Farben treten in nur drei Fällen auf (C. C. Jensen, Danish Wind Export Association, Mita-Teknik A/S) und spielen somit keine bedeutende Rolle als Indikator von dänischer Herkunft. In gleicher Weise wird auch die dänische Flagge in nur drei Fällen benutzt, dafür aber als auffälliges Positionierungsinstrument. Bei CT Offshore A/S wird unter der Überschrift Our Values ein Foto gezeigt, auf dem die dänische Flagge auf See weht. Unter dem Foto werden die Unternehmenswerte aufgelistet: Safety above all, Best in class, Reliable partner, Team spirit und Innovation and adaptability. Bei der Transportfirma Torben Rafn \& Co. A/S und bei Danish Yachts A/S ist die Flagge ein zentraler Teil des Logos.

Bemerkenswert erscheint, dass auf sieben der 41 Websites, die den nationalen Ursprung thematisieren, dieser mit einer internationalen Orientierung kombiniert wird. So schreibt z. B. DEIF A/S: DEIF Wind Power Technology is an independent business area of DEIF A/S, which was established in Denmark in 1993 and is a global supplier of green, safe and reliable control solutions. Weitere Beispiele für die Kombination national-international sind die Website des Unternehmens Poly Tech A/S, das sich als an international Danish owned company präsentiert, sowie die folgende Formulierung von Gibo Plast A/S: en international dansk baseret børsnoteret koncern [,ein internationaler dänisch basierter börsennotierter Konzern'] und schließlich die Selbstdarstellung von Hove A/S als a privately held global company with the headquater located in Copenhagen, the capital of Denmark. Djuma Industry A/S verknüpft einen internationalen Hintergrund mit einer soliden Fundierung in der Region Ostjütland; dabei handelt es sich zwar um einen regionalen Verweis, aber immerhin ist auch dies Ausdruck einer zweiteiligen Unternehmensidentität. Über die nationalen Identitätskomponenten hinaus signalisieren diese Beispiele auch, dass nationale Eigentümerverhältnisse von Bedeutung sind.

Was die Sprachen auf den dänischen Websites betrifft, steht in vier Fällen die Website allein in dänischer Sprache zur Verfügung, was als markanter Indikator für die dänische Herkunft betrachtet werden kann. Dabei fragt man sich, ob sich diese Unternehmen ausschließlich an dänischsprachige Besucher und Aussteller auf der Hamburger Messe wenden, oder ob sie durch andere Kanäle als über ihre Webpräsenz Empfänger in anderen Sprachen als Dänisch ansprechen. Abgesehen von diesen vier Websites und einer weiteren, die allein Finnisch, Schwedisch und Dänisch anbietet, sind sämtliche Websites in englischer Sprache vorhanden. Über die englische Sprache hinaus wird auf vielen Websites eine ganze Vielfalt von Sprachen angeboten, vor allem Deutsch (25), Schwedisch, Spanisch, Polnisch, Russisch und Chinesisch. Als Beispiel für diese Sprachenvielfalt dient das Unternehmen C. C. Jensen A/S. Es hat neun verschiedene Flaggen auf seiner Website, was nicht nur auf ein international tätiges Un- 
ternehmen deutet, sondern auch auf eine Wertschätzung von Sprachenvielfalt. Die Angabe von Sprachenwahl erfolgt entweder durch Buchstaben, z. B. Deutsch oder als Abkürzung DE, oder anhand einer Nationalfahne. Im dänischen Teil des Textkorpus tritt auf 22 Websites die dänische Flagge als Indikator für Sprachenwahl auf. In dieser Funktion lässt sich die Flagge jedoch nicht unbedingt als COO-Merkmal kategorisieren, obwohl implizit auf Nationalitäten verwiesen wird. Ein deutlicheres Signal nationaler Zugehörigkeit macht die gewählte Internetdomain aus; hier zeigt die Analyse, dass 41 Unternehmen die Domain $d k$ verwenden.

Bei den restlichen 25 Websites sind keine nationalen Identitätskomponenten zu erkennen. Gemeinsam für diese ,globalisierten“ Websites sind zum einen die Verwendung der Internetdomain com und zum anderen die Verwendung der englischen Sprache. In den meisten Fällen sind diese Websites nur in Englisch, aber es gibt auch partiell Beispiele mit weiteren Sprachen wie z. B. Deutsch oder Chinesisch. Ein Beispiel für eine dementsprechend globalisierte Website ist die von Svendborg Brakes ApS. Das Unternehmen verweist auf sich selbst als The Global Leading Expert und verweist weiter auf our global mindset and vision.

\section{Zusammenfassende Bewertung der Ergebnisse}

Die Analyse der 100 deutschen und rd. 100 dänischen Websites von Unternehmen der Cleantech-Branche hat ergeben, dass die Verwendung nationaler Identitätskomponenten in der Unternehmenskommunikation im Globalisierungsprozess nicht verdrängt worden ist. Die Darstellung nationaler Zugehörigkeit als Teil der Unternehmensidentität ist vielfältig und weist auch Unterschiede zwischen den deutschen und dänischen Websites auf. Selten scheint es sich um ein Entweder-oder, d. h. Betonung nationaler Zugehörigkeit oder globalisiertes Selbstverständnis, zu handeln; vielmehr findet sich in den meisten Fällen eine Kombination mehr oder weniger hervorgehobener globaler und nationaler Merkmale der Identitätszugehörigkeit. Für die Bewertung der vorliegenden Befunde wird in Anlehnung an die in Abschnitt 3 vorgeschlagene Skalierung von Nielsen (2005) hier eine Skala zwischen Nationalität und Globalität aufgestellt. Dementsprechend lassen sich die Ergebnisse der Analyse in drei Kategorien einordnen: starke COO-Markierung, schwache COO-Markierung und keine COOMarkierung. Am einen Ende der Skala befinden sich somit Websites, auf denen die nationale Zugehörigkeit als Positionierungsparameter stark ausgeprägt ist. Das andere Ende der Skala belegen Websites, die keine Merkmale nationaler Zugehörigkeit aufweisen und dadurch eine ,globalisierte‘ Identität erkennbar werden lassen. Zwischen diesen beiden Endpunkten befinden sich Websites mit relativ schwachen COO-Markierungen. In diese Kategorie fällt der Großteil der hier untersuchten Websites.

Die nationale Zugehörigkeit betonen 41 dänische und 34 deutsche Websites in der Weise, dass sie auf das jeweilige Land explizit verweisen. Dafür werden jedoch weder die Nationalfahnen noch die Nationalfarben in bemerkenswertem Umfang eingesetzt. Die Verweise erfolgen hauptsächlich durch die Nennung von Dänemark bzw. Deutschland als (bewusste) Standort(wahl). Vergleicht man dabei den Anteil dieser national geprägten Websites an den beiden Teilkorpora ist keine große Abweichung festzustellen, aber betrachtet man die Art und Weise, wie diese Prägung konkretisiert wird, werden Unterschiede deutlich. Auf den dänischen Websites wird meist nur angedeutet, was diese nationale Zugehörigkeit konnotiert. So werden sporadisch Ingenieurskunst bzw. grüne Technologie, Expertise und Innovation in Zusammen- 
Nationale Identitätskomponenten in der Unternehmenskommunikation

hang mit der dänischen Herkunft gesetzt. Die deutschen Websites bringen dagegen deutlicher zum Ausdruck, was sie mit der deutschen Herkunft verbinden, so z. B. Qualität, Erfahrung und Zuverlässigkeit. Dieser Unterschied lässt sich vielleicht durch eine Tendenz in Deutschland zur Direktheit und eine Tendenz in Dänemark zur Indirektheit erklären. Bemerkenswert ist außerdem, dass die genannten Merkmale mit den Ergebnissen älterer Studien übereinstimmen (cf. Abschnitt 3); es ergibt sich also hier kein Beleg für geänderte Positionierungsparameter in der Cleantech-Branche.

In dieser Untersuchung wurde außerdem deutlich, dass Slogans eine sehr geringe Rolle in der Markierung nationaler Identität in der Cleantech-Branche spielen. Dieses Ergebnis unterscheidet sich von den Befunden von Nielsen (2005), während die Internetdomain und die integrierte Thematisierung der Nationalität eine deutlichere Rolle spielen. Die Skalierung von Nielsen (2005) erscheint also teilweise verwendbar, es treten aber Unterschiede auf, die auf Branchenspezifika beruhen könnten.

Obwohl ein bedeutender Teil der Websites auf ihre nationale Zugehörigkeit verweist, lässt sich die Taxonomie von Jaffe/Nebenzahl (2006) nicht direkt auf die Websites übertragen. Zwar finden sich die klassische COO-Auffassung und die klassische ,Made in'-Bezeichnung vor, die bei Jaffe/Nebenzahl Country of Origin und Made-in Country entspricht. Daneben kommen jedoch auch zwei weitere Kategorien vor, die bei Jaffe/Nebenzahl nicht auftreten; Quality ensured in Germany sowie Danish owned/dansk baseret. Diese Elemente drücken keine Information über die eigentlichen Design- oder Produktionsorte aus, sondern im ersten Fall, dass die Arbeit mit der Qualitätssicherung in Deutschland erfolgt, und im zweiten Fall, dass auf die Nationalität der Eigentümerschaft Wert gelegt wird. Besonders die letzte Kategorie ist bemerkenswert, weil offen bleibt, was damit signalisiert werden soll. Diese beiden Kategorien könnten folglich der Taxonomie von Jaffe/Nebenzahl hinzugefügt werden.

In die Kategorie der schwachen COO-Markierung fallen diejenigen Websites, die ihre nationale Zugehörigkeit eher implizit angeben. Hierzu gehören die Websites mit den Internetdomains de bzw. $d k$, die als Signal eines Herkunftslands angesehen werden können, auch wenn Internetdomains nicht unbedingt als Positionierungsparameter eingesetzt werden. Dies trifft für 41 dänische und 55 deutsche Websites zu. Ferner umfasst die Zwischenkategorie die Websites, die Nationalfahnen (statt Buchstaben) als Anzeiger für Sprachenwahl verwenden. Auch wenn dieser im Web weit verbreitete Gebrauch von Nationalfahnen nicht vorrangig als Symbol von Nation benutzt wird und auch kaum nationale Zugehörigkeit betont, zeugt dieser Gebrauch von einem an die Einheit Nation geknüpften Bewusstsein beim Absender.

Die dritte Kategorie umfasst die Websites ohne COO-Markierung. Im dänischen Teil des Textkorpus finden sich 25 Websites, die ihre Website ausschließlich auf Englisch anbieten und die Internetdomain com benutzen. Im Vergleich dazu trifft dies für nur sieben deutsche Websites zu. Dieser Unterschied legt den Schluss nahe, dass viele dänische Unternehmen vermuten, dass alle über Englisch als Lingua franca kommunizieren können und dass dies ausreicht, um erfolgreiche und dauerhafte Geschäftsbeziehungen zu etablieren. Bei vielen Unternehmen werden jedoch mehrere Sprachen zur Wahl gestellt. Nur vier dänische Unternehmen und nur elf deutsche bieten die Informationen ihrer Website nicht in englischer Sprache an. Der signifikante Unterschied in der Verwendung des Englischen hängt auch zweifellos mit den unterschiedlichen Größen des deutschsprachigen Raums (ca. 100 Mio. Einwoh- 
ner) und des dänischsprachigen Raums (ca. 5 Mio.) zusammen. Aufgrund der beiden Tendenzen im Zuge der Globalisierung (cf. Abschnitt 2) lassen sich sowohl die Sprachenvielfalt, die in dieser Untersuchung gefunden wurde, als auch die Wahl von Englisch als alleinige Sprache als eine Beeinflussung durch die Globalisierung betrachten.

Wie früher erwähnt, stellt die Betonung bzw. Nicht-Betonung nationaler Zugehörigkeit keinen alleinstehenden Positionierungsparameter dar. Ein Blick auf andere bedeutungstragende Positionierungsparameter im vorhandenen Textkorpus hat gezeigt, dass die folgenden vier Gruppen von Parametern häufig auftreten: zum einen Aussagen zu Qualität, Sicherheit und Flexibilität, zum anderen Erfahrung des Unternehmens (cf. Kiely et al. 2001: 36: „Zeitraum des Wohnens"), zum dritten Internationalität bzw. globale Tätigkeit und zum vierten Marktführerschaft. Diese Parameter kommen jedoch so oft vor, dass sie keine Differenzierung bewirken, sondern eher als Selbstverständlichkeit zu betrachten sind.

Nach dieser Untersuchung stellt sich eine weiterführende Frage, auf die in diesem Beitrag allerdings keine Antwort gegeben werden soll: Es wäre ebenfalls interessant, die Rolle der regionalen oder lokalen Herkunft bzw. Identität zu untersuchen, und dadurch u. a. festzustellen, ob Globalisierungstendenzen Spuren einer Verschiebung von einer nationalen Identität auf eine eher regionale Identität hinterlassen haben, sowie ob eine solche eventuelle Verschiebung parallel in dänischen und deutschen Unternehmenswebsites zu finden ist oder ob sich diesbezügliche Unterschiede zwischen den Identitätsauffassungen der Unternehmen in den beiden Ländern geltend machen.

Zweck des Beitrags war es zu untersuchen, inwieweit sich die Globalisierung in der Unternehmenskommunikation niederschlägt. Die dargestellten Unternehmensidentitäten nehmen wiederum Einfluss auf das gesellschaftliche Leben im Allgemeinen, nicht zuletzt weil die Menschen in den Unternehmen von ihrer Umwelt geprägt sind und diese auch mitprägen. Auf Basis der hier gefundenen nationalen Identitätskomponenten kann festgestellt werden, dass der Nationalitätsbezug weiterhin Bedeutung hat und als wertvoller Positionierungsparameter eingeschätzt wird, auch in einer relativ jungen Branche wie der Cleantech-Branche. Inwieweit dies als Tendenz zur verstärkten Aufmerksamkeit für (national)kulturelle Unterschiede zu bewerten ist, lässt sich hier nicht beantworten, dafür sind weitere Studien erforderlich.

\section{Literatur}

Bamberg, Michael/De Fina, Anna/Schiffrin, Debora (2011): "Discourse and Identity Construction". In: Schwartz, Seth J./Luyckx, Koen/Vignoles, Vivian L. (eds.): Handbook of Identity Theory and Research. New York, Springer: 177-200.

Beck, Ulrich (1998): Was ist Globalisierung? Irrtümer des Globalismus - Antworten auf Globalisierung. Frankfurt a. M.: Suhrkamp.

Birkigt, Klaus/Stadler, Martinus/Funck, Hans Joachim (eds.) (2002): Corporate Identity. Grundlagen - Funktionen - Fallbeispiele. Landsberg/Lech: Moderne Industrie.

Brock, Ditmar (2008): Globalisierung - Wirtschaft, Politik, Kultur, Gesellschaft. Wiesbaden: VS Verlag für Sozialwissenschaften.

Devine, Tom/Logue, Paddye (eds.) (2002): Being Scottish: Personal Reflections on Scottish Identity Today. Edinburgh: Polygon. 
Nationale Identitätskomponenten in der Unternehmenskommunikation

Djursaa, Malene/Kragh, Simon/Møller, Jan Holm (1991): „Det nationale image i eksportmarkedsføringen“. Ledelse og Erhvervsøkonomi 55: 123-132.

Douglas, Fiona (2009): Scottish Newspapers, Language and Identity. Edinburgh: Edinburgh University Press.

Eksportrådet (2014): Sektoranalyse - Miljø \& Energi. Tyskland. Kopenhagen: Udenrigsministeriet.

Erhvervs- og Vækstministeriet (2015): Grøn omstilling og vaekst. www.evm.dk/arbejdsomraader/vaekst-og-konkurrenceevne/groen-omstilling-og-vaekst [20.04.2015].

Gilroy, Paul (1997): "Diaspora and the Detours of Identity". In: Woodward, Kathryn (ed.): Identity and Difference. London, Sage Publications/Open University Press: 299-343.

Giese, Ernst/Mossing, Ivo/Schröder, Heike (2011): Globalisierung der Wirtschaft - Eine wirtschaftsgeographische Einführung. Paderborn: Schöningh.

Gilroy, Paul (2000): Between Camps: Nations, Culture and the Allure of Race. London: Lane.

Hall, Edward T. (1976): Beyond Culture. Garden City/NY: Doubleday.

Hatch, Mary Jo/Schultz, Majken (1997): "Relations between organisational culture, identity and image". European Journal of Marketing 31/5: 356-365.

Hofstede, Geert (1980): Culture's Consequences: International Differences in Work-Related Values. Beverly Hills: Sage Publications.

Head, David (1988): “Advertising Slogans and the 'Made in' Concept”. International Journal of Advertising 7: 237-252.

Herbst, Dieter Georg (2012): Corporate Identity. Aufbau einer einzigartigen Unternehmensidentität. Berlin: Cornelsen.

Ind, Nicholas (1992): The Corporate Image. London: Page.

Jaffe, Eugene D./Nebenzahl, Israel D. (2006): National Image \& Competitive Advantage. The Theory and Practice of Place Branding. Kopenhagen: Copenhagen Business School Press.

Jenkins, Richard (2004): Social Identity. 2. Auflage. London/New York: Routledge.

Jenkins, Richard (2014): Social Identity. 4. Auflage. London/New York: Routledge.

Jensen, Iben (2013): Grundbog i kulturforståelse. Kopenhagen: Samfundslitteratur.

Kabir, Nahid Afrose (2013): Young American Muslims: Dynamics of Identity. Edinburgh: Edinburgh University Press.

Kiely, Richard et al. (2001): "Markers and Rules of Scottish National Identity". Sociological Review 49/1: 33-55.

Kückelhaus, Andrea (1998): Public Relations: Die Konstruktion von Wirklichkeit. Kommunikationstheoretische Annäherung an ein neuzeitliches Phänomen. Opladen: Westdeutscher Verlag.

Meffert, Heribert/Burmann, Christoph/Kirchgeorg, Manfred (2012): Marketing. Grundlagen marktorientierter Unternehmensführung. Wiesbaden: Gabler.

Melewar, T.C./Jenkins, Elisabeth (2002): "Defining the Corporate Identity Construct". Corporate Reputation Review 5/1: 76-90.

Melewar, T.C./Karaosmanoglu, Elif (2006): "Seven dimensions of corporate identity. A categorisation from the practitioners' perspectives". European Journal of Marketing 40/7-8: 846-869. 
Melewar, T.C./Wooldridge, Arian (2001): "The dynamics of corporate identity". Journal of Communication Management: An International Journal 5/4: 327-340.

Mole, Richard C.M. (2007): "Discursive Identities/Identity Discourses and Political Power". In: Mole, Richard C.M. (ed.): Discursive Constructions of Identity in European Politics. Basingstoke, Macmillan: 1-24.

Nielsen, Martin (2005): "Made in Denmark, sold in Germany - zur Verwendung des Countryof-Origin-Prinzips im dänisch-deutschen Kontext". In: Janich, Nina (ed.?): Unternehmenskultur und Unternehmensidentität. Wirklichkeit und Konstruktion. Wiesbaden, Deutscher Universitätsverlag: 155-169.

Niss, Hanne (1994): Made in Denmark. Nationalitetens betydning i international markedsføring. Aalborg: Aalborg Universitetsforlag.

Niss, Hanne (1996): "Country of Origin Marketing over the Product Life Cycle". European Journal of Marketing 30/3: 6-22.

Papadopoulos, Nicholas (1993): "What Product and Country Images Are and Are Not". In: Papadopoulos, Nicholas/Heslop, Louise (eds.): Product-Country Images. Impact and Role in International Marketing. Binghamton, International Business Press: 3-38.

Rowlinson, Michael/Procter, Stephen (1999): "Organisational culture and business history". Organisational Studies 20/3: 369-396.

Saldsieder, Kai Alexander/Saldsieder, Nina (2010): „Wettbewerbsdifferenzierung durch Lizenzen“. In: Görg, Ulrich (ed.): Erfolgreiche Markendifferenzierung. Strategie und Praxis professioneller Markenprofilierung. Wiesbaden, Gabler: 181-206.

Singh, Nitish/Pereira, Arun (2005): The Culturally Customized Web Site. Customizing Web Sites for the Global Marketplace. Burlington/Oxford: Elsevier.

Solvang, Anja (2014): „Tyskland tilbage på vækstsporet“. Eksportfokus 1/2014: 14-19.

Varey, Richard/Lewis, Barbara (2000): Internal Marketing: Directions of Management. London: Routledge.

$\mathrm{WE}=$ Ausstellerverzeichnis der WindEnergy Hamburg (2014). http://we14.mediahmc.de/catalog/index.cfm [20.04.2015].

Wikipedia (2015): Top-Level-Domain. http://de.wikipedia.org/wiki/Top-Level-Domain [20.04.2015]. 